\title{
Increased soluble ICAM-1 concentration and impaired delayed-type hypersensitivity skin tests in patients with chronic liver disease
}

\author{
M Pirisi, D Vitulli, E Falleti, C Fabris, G Soardo, M Del Forno, P Bardus, F Gonano, \\ E Bartoli
}

\begin{abstract}
Aims/background-Soluble ICAM-1 may act as an antagonist of the membrane bound form, which is essential for the adhesion of leucocytes to endothelial cells. The aim of this study was to investigate whether the presence of high concentrations of soluble ICAM-1 are related to the impairment of delayed-type hypersensitivity reactions.

Methods-The study population comprised 73 patients ( 53 men and 20 women) with chronic liver disease (19 with chronic hepatitis, 36 with cirrhosis and 18 with hepatocellular carcinoma), and 21 agematched controls ( 11 men and 10 women). Serum soluble ICAM-1 was measured using an enzyme immunoassay. Skin tests for seven different antigens (tetanus, diphtheria, streptococcus group C, tuberculin, Candida, tricophyton, and proteus) were considered positive when diameters $\geq 2 \mathrm{~mm}$ were recorded; the diameters of positive tests were added to calculate a cumulative score.

Results-Patients with chronic liver disease had fewer positive skin tests (median 2) and a lower cumulative score (median 7) than controls (median 3 and 12, respectively). Multivariate analysis suggested the existence of an independent association between alkaline phosphatase and anergy to skin tests and between soluble ICAM-1 concentrations and the cumulative score.

Conclusions-The strong association observed between increased soluble ICAM-1 concentrations and impairment of delayed-type hypersensitivity skin tests suggests that soluble ICAM-1 may be implicated in the immune depression seen in patients with chronic liver disease. (F Clin Pathol 1997;50:50-53)
\end{abstract}

Dipartimento di Patologia e Medicina Sperimentale e Clinica, Università di Udine, Udine, Italy

Correspondence to: Dr Mario Pirisi, Cattedra di Medicina Interna, Universita degli Studi, Piazzale Santa Maria della Misericordia 1, 33100 Udine, Italy.

Keywords: delayed-type hypersensitivity; chronic liver disease; adhesion molecules.

Delayed-type hypersensitivity (DTH) skin tests have long been used as a rapid, inexpensive, and readily available method for assessing $T$ lymphocyte function in vivo. In fact, when a recall antigen is injected into the skin, antigen specific $T$ cells are activated at that site and lymphokines are produced. As a result, macrophages and lymphocytes migrate to the site over the next 48 hours and the typical signs of a DTH appear.' Although skin tests are convenient, there are several potential pitfalls in the interpretation of their results: to mention but two, the DTH reaction tends to wane with age $^{2}$ and the proportion of subjects who are positive to non-ubiquitous antigens varies widely according to the frequency of exposure to those antigens in the reference population. Besides, the results of DTH skin tests need to be interpreted with caution in patients with chronic liver disease as, for unclear reasons, the incidence of positive DTH skin tests in these patients is low. ${ }^{3}$ One possible explanation which has been proposed for this finding in patients with alcoholic cirrhosis is the reduced numbers of circulating $\mathrm{T}$ lymphocytes. ${ }^{4}$ Others have proposed that plasma inhibitors of $\mathrm{T}$ lymphocyte function, still to be identified, may contribute to their anergy.

The recently described circulating form of intercellular adhesion molecule-1 (sICAM-1) ${ }^{6}$ may act as an antagonist of the membrane bound form, which is essential for leucocyte adhesion to endothelial cells. sICAM-1 might hinder adhesion by competing for the integrin ligand of ICAM- $1^{7}$; in a similar way, soluble interleukin-2 receptor abrogates the proliferation of activated $T$ cells by competing for interleukin-2 with the interleukin-2 receptor. ${ }^{8}$ Patients with liver diseases of different aetiologies have increased sICAM-1 concentrations, which correlate strictly with indexes of cholestasis and of functioning hepatic mass. ${ }^{9-11}$ One may ask, therefore, whether sICAM-1 plays a role in the immune deficiency which is often observed in patients with chronic liver diseases. Thus, in the present paper we analysed sICAM-1 concentrations, among other parameters, to elucidate the factors associated with reduced skin test responsiveness in patients with chronic liver disease.

\section{Methods}

In total, 94 anti-HIV negative subjects were enrolled in the study; 73 patients (53 men and 20 women; mean (SD) age 58.4 (10.5), range 23-81 years) had chronic liver diseases. Their clinical and demographic characteristics are outlined in table 1 . Twenty one healthy subjects (11 men and 10 women; age 58.4 (9.8), range 39-75 years) served as controls. Chronic hepatitis was always confirmed by biopsy. The diagnosis of cirrhosis was either histological, based on the examinination of a 
liver biopsy specimen, or clinical, on the basis of hypoalbuminaemia, hypergammaglobulinaemia, ascites, and signs of portal hypertension. The diagnosis of hepatocellular carcinoma was based on suggestive diagnostic imaging or high $(>400 \mu \mathrm{g} / \mathrm{l})$ concentrations of serum $\alpha$-fetoprotein, and confirmed in all instances by biopsy or at necropsy. None of the subjects had evidence of active tuberculosis. Informed consent was obtained from all participants in the study, which was conducted in strict observance of the principles of the Declaration of Helsinki.

\section{STUDY PROTOCOL}

A pre-loaded, multidose applicator (Multitest IMC, Pasteur Mérieux, France) for seven different antigens (tetanus, diphtheria, streptococcus group C, tuberculin, Candida, tricophyton, and proteus) plus a glycerin control test was used for skin testing. Two days later, the results of the skin tests were recorded, averaging the readings taken by two independent observers, who determined the diameter of the induration by taking measurements across the lesion at 90 degrees to each other. A diameter $\geq 2 \mathrm{~mm}$ was considered indicative of a positive test. A cumulative score (C-score) was obtained by summing the diameters of all positive tests.

Fasting blood samples were drawn on the day of the skin tests. Aliquots of serum were kept frozen at $-80^{\circ} \mathrm{C}$ pending measurement of the sICAM-1 concentration, by means of an enzyme linked immunoassay (British Biotechnology Products Ltd, UK). This assay, which incorporates a mouse monoclonal antibody directed against ICAM-1, has a typical sensitivity of $<2.5 \mu \mathrm{g} / \mathrm{l}$, with an intra-assay pre-

Table 1 Demographic and clinical characteristics of the patients studied. Data are expressed as mean (SD) for numerical variables and as frequencies for categorical variables

\begin{tabular}{lcll}
\hline & $\begin{array}{l}\text { Chronic hepatitis } \\
(n=19)\end{array}$ & $\begin{array}{l}\text { Cirrhosis } \\
(n=36)\end{array}$ & $\begin{array}{l}\text { Hepatocellular } \\
\text { carcinoma } \\
(n=18)\end{array}$ \\
\hline Age (years) & $50.3(11.2)$ & $59.3(8.2)$ & $65.3(8.3)$ \\
Sex & 12 & 27 & 14 \\
$\quad$ Male & 7 & 9 & 4 \\
$\quad$ Female & 1 & 1 & 1 \\
Aetiology & 18 & 19 & 8 \\
$\quad$ Hepatitis B & - & 15 & 1 \\
$\quad$ Hepatitis C & - & 1 & $37.6(5.2)$ \\
$\quad$ Alcohol & $43.6(2.4)$ & $38.5(5.3)$ & $43.9(86.9)$ \\
$\quad$ Others, indeterminate & $12.7(3.4)$ & $28.4(25.6)$ & $55 \quad(43)$ \\
Albumin (g/l) & $112(79)$ & $74 \quad(68)$ & \\
Bilirubin $(\mu$ mol/l) & & & \\
Alanine aminotransferase (U/l) & & \\
\hline
\end{tabular}

Table 2 Rates of response to delayed-type hypersensitivity skin tests in patients and controls. Results are expressed as frequencies (\%)

\begin{tabular}{lcccc}
\hline & $\begin{array}{c}\text { Controls } \\
(n=21)\end{array}$ & $\begin{array}{l}\text { Chronic } \\
\text { hepatitis } \\
(n=19)\end{array}$ & $\begin{array}{l}\text { Cirrhosis } \\
(n=36)\end{array}$ & $\begin{array}{l}\text { Hepatocellular } \\
\text { carcinoma } \\
(n=18)\end{array}$ \\
\hline Anergic & $1(5)$ & $3(16)$ & $5(14)$ & $4(22)$ \\
Single positive & $4(19)$ & $3(16)$ & $11(31)$ & $2(11)$ \\
Two or more positive & $16(76)$ & $13(68)$ & $20(56)$ & $12(67)$ \\
Tetanus & $13(62)$ & $12(63)$ & $16(44)$ & $7(39)$ \\
Diphteria & $9(43)$ & $5(26)$ & $5(14)$ & $7(39)$ \\
Streptococcus & $3(14)$ & $2(11)$ & $6(17)$ & $0(0)$ \\
Tuberculin & $13(62)$ & $11(58)$ & $19(53)$ & $12(67)$ \\
Candida & $11(52)$ & $7(37)$ & $10(28)$ & $8(44)$ \\
Trycophiton & $3(14)$ & $3(16)$ & $7(19)$ & $1(6)$ \\
Proteus & $4(19)$ & $2(11)$ & $2(6)$ & $3(17)$ \\
\hline
\end{tabular}

Table 3 Univariate regression analysis between the number of positive delayed-type hypersensitivity skin tests and selected variables

\begin{tabular}{lcl}
\hline Variable & Spearman $\rho$ & p value \\
\hline Age & 0.094 & $\mathrm{NS}$ \\
Leucocyte count & 0.054 & $\mathrm{NS}$ \\
Lymphocyte count & 0.170 & $\mathrm{NS}$ \\
Erythrocyte sedimentation rate & -0.297 & $<0.005$ \\
Total bilirubin & -0.198 & $<0.10$ \\
Aspartate aminotransferase & -0.309 & $<0.005$ \\
Alkaline phosphatase & -0.458 & $<0.001$ \\
Albumin & 0.205 & $<0.005$ \\
Total cholesterol & 0.267 & $<0.01$ \\
Triglycerides & 0.208 & $<0.05$ \\
$\gamma$-globulin & -0.276 & $<0.01$ \\
sICAM-1 & -0.386 & $<0.001$ \\
\hline
\end{tabular}

cision ranging from 5.4 to $8.3 \%$ and an inter-assay precision ranging from 7.1 to $8.8 \%$.

\section{STATISTICAL ANALYSIS}

The normality of data was checked by the Shapiro and Wilk W test. When appropriate, those parameters which departed significantly from a normal distribution were logarithmically converted; an observed value of 0 was transformed to $\log _{10} 1$. Occasional missing values were estimated by regression on all available variables. Mann-Whitney rank sum tests (two-tailed) were used to verify the existence of differences in the results of the skin tests between patients with chronic liver disease and controls. Correlation coefficients were calculated by parametric and non-parametric methods to relate the results of skin tests to the variations of continuous variables. The existence of an association between anergy to DTH skin tests and specific categorical variables was verified by use of Pearson $\chi^{2}$ tests. Those numerical or categorical variables significantly associated with the results of skin tests on univariate analysis were tested on multivariate analysis using a stepwise approach. All statistical tests were performed by means of the BMDP statistical software package, release 7.0 (Statistical Software, Ireland).

\section{Results}

The rates of response to each single antigen in the studied population, grouped according to the diagnostic categories considered, are given in table 2. Although patients with chronic liver disease did not present a statistically higher rate of anergy to DTH skin tests compared with controls $\left(12 / 73 v 1 / 21\right.$, Pearson $\chi^{2} 1.87$, NS), the number of positive DTH skin tests in the former was lower (median 2, 95\% confidence interval (CI) $1-2 v 3,95 \%$ CI $2-3$; p < 0.05 , Mann-Whitney test). There were no statistically significant differences in the rate of anergy according to sex or aetiology of liver disease. The results of univariate regression analysis between the number of positive skin tests and a panel of variables is presented in table 3. Stepwise logistic regression analysis of those variables found to be significant on univariate analysis indicated that alkaline phosphatase was the only factor associated independently with anergy to DTH skin tests (improvement $\chi^{2}=7.97, p=0.005$ ).

Figure 1 shows the scatterplot of individual $\mathrm{C}$-score values. Patients with chronic liver dis- 


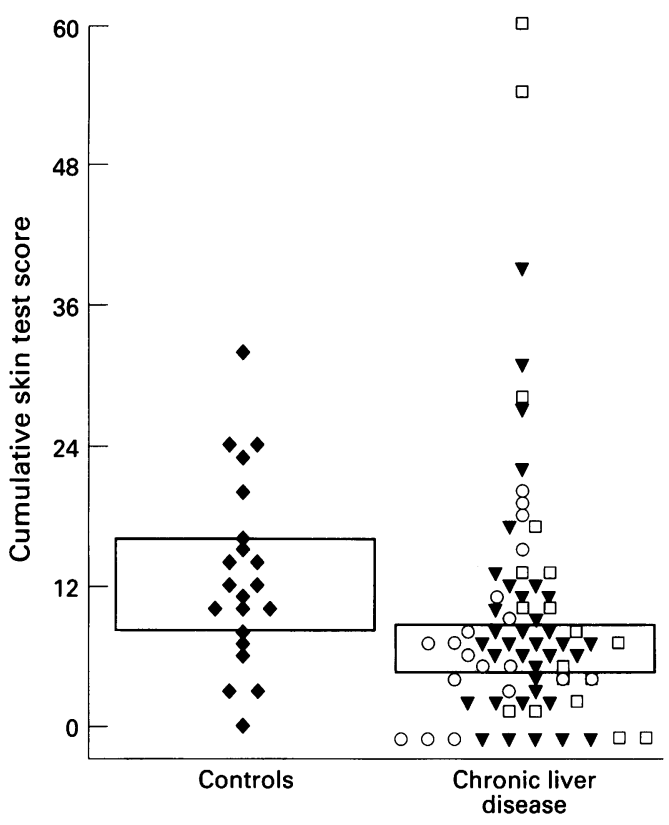

Figure 1 Scatterplot of individual cumulative skin test scores in control subjects (diamonds) and in patients with chronic liver disease (non-cirrhotic liver disease: circles; cirrhosis: triangles; hepatocellular carcinoma: squares). The boxes represent the $95 \%$ confidence intervals in each group.

Table 4 Univariate regression analysis between cumulative skin test score and selected variables

\begin{tabular}{lrl}
\hline Variable & $r$ & \multicolumn{1}{c}{$p$ value } \\
\hline Age & 0.182 & $<0.10$ \\
Leucocyte count & 0.024 & $\mathrm{NS}$ \\
Lymphocyte count & 0.213 & $<0.05$ \\
Erythrocyte sedimentation rate & -0.238 & $<0.05$ \\
Total bilirubin & -0.300 & $<0.005$ \\
Aspartate aminotransferase & -0.322 & $<0.005$ \\
Alkaline phosphatase & -0.392 & $<0.001$ \\
$\gamma$-glutamyl transpeptidase & -0.127 & $\mathrm{NS}$ \\
Albumin & 0.311 & $<0.005$ \\
Total cholesterol & 0.273 & $<0.01$ \\
Triglycerides & 0.165 & $\mathrm{NS}$ \\
$\gamma$-globulin & -0.302 & $<0.005$ \\
sICAM-1 & -0.425 & $<0.001$ \\
\hline
\end{tabular}

ease had a lower C-score compared with controls (median 7, 95\% CI 5-8 v 12, 95\% CI $8-16 ; \mathrm{p}<0.01$, Mann-Whitney test). Table 4 presents the results of univariate regression analysis between C-score and a selection of numerical variables. Among those variables statistically associated with C-score on univariate analysis, sICAM-1 was the strongest independent predictor of the C-score value on stepwise multiple regression analysis (change in $r^{2}=0.18$ ), followed by age (change in $r^{2}=$ 0.04).

\section{Discussion}

The first histological changes in the DTH reaction are observed four to eight hours after injection of the antigen and are represented by dermal vasodilation and oedema, with a cell infiltrate around the deep dermal vessels. In recent years, it has been appreciated that venular endothelial cells play a crucial role in regulating this infiltrate, which is mainly lymphohistiocytic. In fact, it has been shown that, in response to cytokines and in an orderly time dependent fashion, the expression of molecules adhesive for circulating leucocytes increases on endothelial cells. The first to be expressed, and the first to decline following cytokine stimulation, is E-selectin. The expression of ICAM-1 occurs more slowly, but is more prolonged. ${ }^{12}$ The interaction between endothelial cell ICAM-1 and its ligand, lymphocyte function activation antigen-1 (LFA-1), expressed on circulating neutrophils, monocytes and lymphocytes, is reputedly essential for the promotion of leucocyte migration from the circulation into tissue.

In agreement with the results of others, ${ }^{34}$ we found that patients with chronic liver disease have a reduced response to DTH skin tests compared with age-matched controls. Although impaired DTH reactivity seemed to be associated with several variables on univariate analysis, on multivariate analysis the serum concentrations of sICAM-1 and of alkaline phosphatase were the strongest independent predictors of the DTH skin test C-score and of an anergic status, respectively. The serum concentrations of both alkaline phosphatase and sICAM-1 are strictly related to cholestasis, confirming that cholestasis is major pathophysiological event associated with the impaired DTH reactivity of patients with chronic liver disease. It should be noted that, although age was also found to be an independent predictor of C-score, the association between age and C-score was entirely dependent on the results of tuberculin testing. When tuberculin tests were excluded, the association between age and C-score disappeared completely (data not shown), suggesting that it was due to age related differences in exposure to Mycobacterium tuberculosis. ${ }^{13}$

The reasons why cell-mediated immunity is depressed in most forms of liver disease are unknown. These patients might share some common factor with other anergic subjects, such as advanced age and poor nutritional status. However, a specific relation with liver damage is also probable, as similar changes are seen in rats with induced cirrhosis. ${ }^{14}$ Immunohistochemical studies have shown clearly that ICAM-1, which is restricted to cells lining sinusoids and portal vessels in the normal liver, is expressed on hepatocytes in patients with hepatitis. ${ }^{15}$ sICAM-1, which results from proteolytic cleavage of membrane bound ICAM-1 close to the cell membrane, is significantly increased in patients with acute viral- and druginduced hepatitis, chronic active hepatitis and active cirrhosis. ${ }^{10}$ If it were shown that SICAM-1 (which is excreted into the bile) ${ }^{16}$ acts as an antagonist of membrane bound ICAM-1, it will become an attractive candidate for the down regulation of immune function in patients with chronic liver disease.

The serum concentrations of both alkaline phosphatase and SICAM-1 are strictly related to cholestasis, which therefore seems to be the major pathophysiological event associated with the impaired DTH reactivity of patients with chronic liver disease. In this regard, it should be pointed out that in the rat a defective acute inflammatory response is caused, at least in part, by a constituent of cholestatic plasma that is antiadhesive to neutrophils. ${ }^{17}$ Moreover, obstructive jaundice causes reduced expression 
of polymorphonuclear leucocyte adhesion molecules and a depressed response to bacterial wall products in vitro, which is abolished by removal of plasma. ${ }^{18}$ Indeed, cholestasis has long been known to be associated with a high incidence of infective complications, for unknown reasons. ${ }^{19}$

Finally, if sICAM-1 exerts a direct immune suppressive action, this should also be observed in conditions not characterised by cholestasis. In this regard, it is interesting to note that raised sICAM-1 concentrations have been found in several extrahepatic diseases, ${ }^{20}$ including rheumatic diseases, diabetes, uraemia, Crohn's disease, and cancer, which are also characterised by anergy.

We conclude that, although the association between increased sICAM-1 concentration and impairment of DTH skin tests cannot be taken as a proof of a cause-effect relation, it does suggest that the circulating form of this adhesion molecule, rather than being simply an inert by-product, may have a pathophysiological role in the immune depression seen in patients with chronic liver disease.

1 Ahmed AR, Blose DA. Delayed-type hypersensitivity skin testing. A review. Arch Dermatol 1983;119:934-45.

2 Battershill JH. Cutaneous testing in the elderly patient. Chest 1980;77:188-9.

3 Thomas HC. Immunologic mechanisms in chronic liver disease. In: Zakim D, Boyer TD, eds. Hepatology: a textboo of liver disease. Philadelphia: WB Saunders, 1990:1114-26.

4 Berenyi MR, Straus B, Cruz D. In vitro and in vivo studie of cellular immunity in alcoholic cirrhosis. Am 7 Dig Dis 1974;19:199-205.

5 Hsu CCS, Leevy CM. Inhibition of PHA-stimulated lymphocyte transformation by plasma from patients with advanced alcoholic cirrhosis. Clin Exp Immunol 1971;8: 749-60.

6 Rothlein R, Mainolfi E, Czajkowski M, Marlin SD. A form of circulating ICAM-1 in human serum. $\mathcal{F}$ Immunol 1991;147:3788-93
7 Gearing AJH, Hemingway I, Pigott R, Hughes J, Rees AJ, Cashman SJ. Soluble forms of vascular adhesion molecules, E-selectin, ICAM-1, and VCAM-1: pathological significance. Ann NY Acad Sci 1992;667:324-31.

8 Rubin LA, Nelson DL. The soluble interleukin-2 receptor: biology, function, and clinical application. Ann Intern Med 1990;113:619-27.

9 Adams DH, Mainolfi E, Burra P, Neuberger JM, Ayres R, Elias E, et al. Detection of circulating intercellular adhesion molecule-1 in chronic liver diseases. Hepatology 1992;16: 810-14.

10 Zöhrens G, Armbrust T, Pirzer U, Meyer Zum Büschenfelde K-H, Ramadori G. Intercellular adhesion molecule-1 concentration in sera of patients with acute and chronic liver disease: relationship to disease activity and cirrhosis. Hepatology 1993;18:798-802.

11 Pirisi M, Falleti E, Fabris C, Soardo G, Toniutto P, Vitulli $\mathrm{D}$, et al. Circulating intercellular adhesion molecule-1 (cICAM-1) concentration in liver disease. Am f Clin Pathol 1994;102:600-4.

12 Bevilacqua MP. Endothelial-leukocyte adhesion molecules. Annu Rev Immunol 1993;11:767-804.

13 Bailey TC, Fraser VJ, Spitznagel EL, Dunagan WC. Risk factors for positive tuberculin skin test among employees of an urban, midwestern teaching hospital. Ann Intern Med 1995;122:580-5.

14 Thomas HC, Singer CRJ, Tilney NL, Folch H, MacSween RNM. The immune response in cirrhotic rats. Antigen distribution, humoral immunity, cell-mediated immunity and splenic suppressor cell activity. Clin Exp Immunol 1976;26: 574-82.

15 Volpes $\mathrm{R}$, van den Oord JJ, Desmet VJ. Immunohistochemical study of adhesion molecules in liver inflammation. Hepatology 1990;12:59-65.

16 Adams DH, Mainolfi E, Elias E, Neuberger JM, Rothlein R. Detection of circulating intercellular adhesion molecule- 1 after liver transplantation: evidence of local release within the liver during graft rejection. Transplantation 1993;55:83-

17 Swain MG, Tjandra K, Kanwar S, Kubes P. Neutrophil adhesion is impaired in a rat model of cholestasis. Gastroenterology 1995;109:923-32.

18 Plusa S, Webster N, Primrose J. Obstructive jaundice causes reduced expression of polymorphonuclear leucocyte adhesion molecules and a depressed response to bacterial wall products in vitro. Gut 1996;38:784-7.

19 Pitt HA, Cameron JL, Postier RG, Gadacz TR. Factors affecting mortality in biliary tract surgery. Am f Surg 1981; 141:66-72.

20 Gearing AJH, Newman W. Circulating adhesion molecules in disease. Immunol Today 1993;14:506-12. 